\title{
Research Article \\ Effect of Tip Clearance on the Internal Flow and Hydraulic Performance of a Three-Bladed Inducer
}

\author{
Yanxia Fu, ${ }^{1}$ Jianping Yuan, ${ }^{2}$ Shouqi Yuan, ${ }^{2}$ Giovanni Pace, ${ }^{3}$ and Luca d'Agostino ${ }^{4}$ \\ ${ }^{1}$ School of Energy and Power Engineering, Jiangsu University, No. 301 Xuefu Road, Zhenjiang, Jiangsu 212013, China \\ ${ }^{2}$ National Research Center of Pumps, Jiangsu University, No. 301 Xuefu Road, Zhenjiang, Jiangsu 212013, China \\ ${ }^{3}$ ALTA S.p.A., 5 via della Gherardesca, Ospedaletto, 56121 Pisa, Italy \\ ${ }^{4}$ Civil and Industrial Engineering, University of Pisa, 8 Via Gerolamo Caruso, 56122 Pisa, Italy \\ Correspondence should be addressed to Yanxia Fu; yanxiafu@ujs.edu.cn
}

Received 7 October 2016; Revised 8 December 2016; Accepted 5 February 2017; Published 8 March 2017

Academic Editor: Zuohua Huang

Copyright (c) 2017 Yanxia Fu et al. This is an open access article distributed under the Creative Commons Attribution License, which permits unrestricted use, distribution, and reproduction in any medium, provided the original work is properly cited.

\begin{abstract}
The influence of the tip clearance on the internal flow and hydraulic performances of a 3-bladed inducer, designed at ALTA, Pisa, Italy, are investigated both experimentally and numerically. Two inducer configurations with different blade tip clearances, one about equal to the nominal value and the other 2.5 times larger, are considered to analyze tip leakage effects. The 3D numerical model developed in ANSYS CFX to simulate the flow through the inducer with 2 different clearances under different operating conditions is illustrated. The internal flow fields and hydraulic performance predicted by the CFD model under different operating conditions are compared with the corresponding experimental data obtained from the inducer tests. As expected, both experimental and numerical results indicate that higher pressure rise and hydraulic efficiency are obtained from the inducer configuration with the nominal tip clearance.
\end{abstract}

\section{Introduction}

The axial inducers, employed upstream of the centrifugal stage of propellant rocket turbopumps, are typically working under the severe conditions that lead to the development of flow instabilities which in turn can seriously degrade the performances of the inducers $[1,2]$. As reported from a lot of open literatures, the blade tip clearance between the impeller and the casing affects the internal flow and usually induces flow instabilities occurring in turbomachinery. Since the vortices are associated with the tip clearance flows, further complexities in the development of cavitation and cavitation induced instabilities will occur, such as backflow vortex cavitation and tip vortex cavitation, which are significantly affected by the inducer casing [3-6]. Furthermore, the hydraulic performance of inducers is sensitive to the tip clearance for clearance/mean blade height ratios higher than $2 \%$ and drops rapidly for larger values of this parameter.

The present study specifically aims at analyzing by comparison with the experimental results the influence of tip clearances on the inducer's predicted internal flows and hydraulic performances. Computations have been carried out by means of the commercial ANSYS CFX 14.5 software package installed at Jiangsu University, Zhenjiang City, China, in the blade high-performance computing clusters system, whose main operational parameters are listed in Table 1. Reference validation experiments have been carried out in the CPRTF (Cavitating Pump Rotordynamic Test Facility) [7] at ALTA, Pisa, Italy, on the three-bladed inducer with two values of the blade tip clearances, designed as described in Pace et al. [8] and manufactured in 7075-T6 aluminum alloy [8].

\section{Numerical Methods}

2.1. Geometrical Data. For predicting the internal flow and hydraulic performances of an inducer by CFD techniques, the computational model of the inducer was combined with inlet and outlet pipes, as shown in Figure 1. The inlet duct was modified with an extended portion about 20 times of the inducer inlet radius, $r_{T i}$, and the outlet duct was also extended in the same way. The main geometrical and operational characteristics of the DAPROT3 inducer were demonstrated in detail by Pace et al. $[8,9]$, respectively. 
TABLE 1: The parameters of blade high-performance computing clusters system.

\begin{tabular}{ll}
\hline CPU & $\begin{array}{l}2 \times \text { Intel Xeon X5650 CPU with 6 physical cores } \\
\text { for each, 2.40 GHz, 12 M three-level buffer } \\
\text { 24 G DDR3 RDIMM, 18 slots, the maximum } \\
\text { memory: 384 G } \\
\text { Memory }\end{array}$ \\
$\begin{array}{l}\text { Hard disk } \\
\text { Blade server } \\
\begin{array}{l}\text { Operating } \\
\text { system }\end{array}\end{array}$ & Dawning Blade Full View Manager System \\
\hline
\end{tabular}

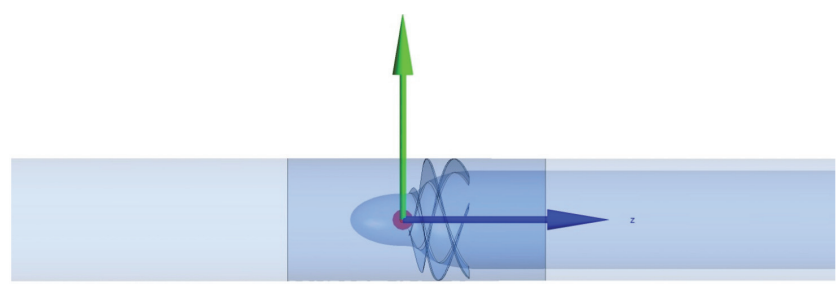

FIGURE 1: Three-dimensional model of the inducer.

2.2. Mesh Generation and Grid Independence Check. Due to their wide adaptability to the complex geometries and reduced time required for the mesh's generation, unstructured tetrahedral cells were applied for the inducer which includes 3 twisted blades and complex inner flow passages by using ANSYS ICEM CFD 14.5. In particular, due to the complexity of the inducer, it was split into a number of component parts which included the BLE, BTE, BS, BP, BTP, nose, and hub for generating mesh as shown in Figure 2. Care was also taken when generating the grids for the inlet and outlet ducts. The final mesh number of the whole computational domains is systematically increased and ranges from 1.74 million to 11.38 million in the present study, as shown in Table 2.

In particular, based on four computational grids (coarser, medium, and finer) summarized in Table 2, the influence of different mesh numbers on the prediction of hydraulic performance of the inducer with the tip clearance of $0.8 \mathrm{~mm}$ operating at a flow coefficient of $\Phi=0.05903$ was especially investigated during the calculating process. As shown in Figure 3 , the prediction of the head coefficient increases slowly with the number of grid elements and remains almost constant with the corresponding experimental data when using the finer grid. The values of Yplus in each computational domain are in the range of 165.32 for the inlet pipe, 168.80 for the inducer, and 198.52 for the outlet pipe, respectively.

\subsection{Governing Equations and Boundary Conditions. ANSYS} CFX is applied based on the Reynolds-averaged NavierStocks equations which solve the momentum, continuity, and turbulence equations for incompressible, turbulent flow of Newtonian fluids in the inducer. The standard $k-\varepsilon$ model in ANSYS CFX falls within this class of models and has become the workhorse of practical engineering flow calculations in the time since it was proposed by Launder and Spalding [1012]. From a turbulence modeling standpoint, the high quality grids are able to provide the calibrated performance. The
TABLE 2: Details of mesh elements.

\begin{tabular}{lcccc}
\hline \multirow{2}{*}{ Computational domains } & \multicolumn{4}{c}{ Mesh elements (million) } \\
& Case 1 & Case 2 & Case 3 & Case 4 \\
\hline All domains & 1.74 & 2.26 & 2.68 & 11.38 \\
\hline
\end{tabular}

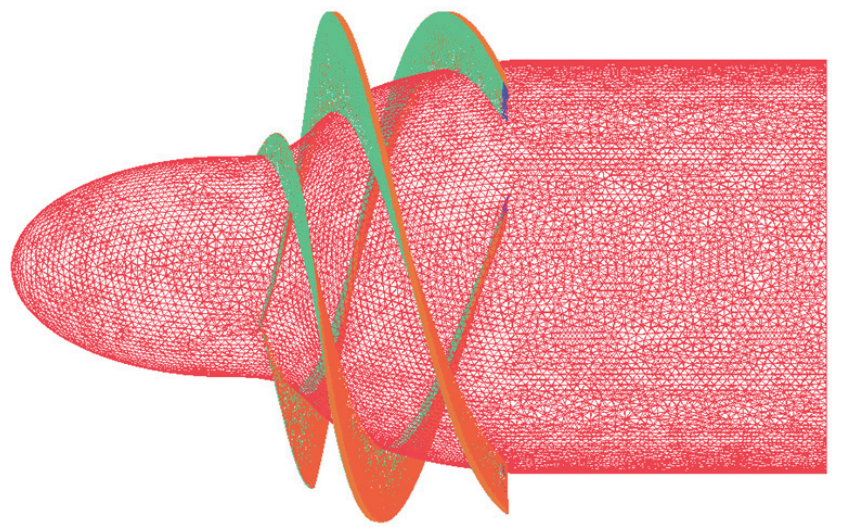

FIgURE 2: Unstructured mesh for the inducer.

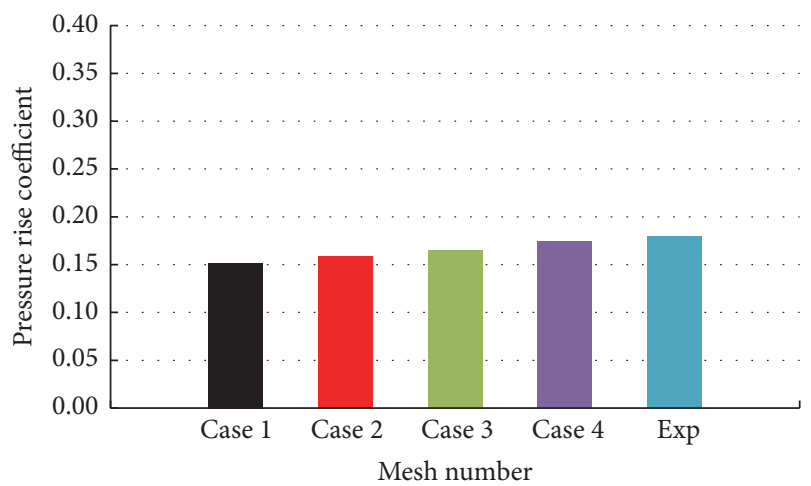

FIGURE 3: The grid independent check for the head of the inducer under $90 \%$ of the design point $(\Phi=0.05903)$.

turbulence model uses the scalable wall function approach to improve robustness and accuracy when the near-wall mesh is very fine. The scalable wall functions allow solution on arbitrarily fine near-wall grids, which is a significant improvement over standard wall functions. As for boundary conditions in the present study, total pressure and a constant mass flow rate condition were applied for the inlet and outlet, respectively.

\section{Experimental Apparatus}

The Cavitating Pump Rotordynamic Test Facility (CPRTF, ALTA S.p.A., Pisa, Italy; Figure 4) is a flexible apparatus that can readily be adapted to conduct experimental investigations on virtually any kind of fluid dynamic phenomena relevant to high-performance turbopumps in a wide variety of alternative configurations (impeller with axial, radial, or mixed flow, with or without an inducer) [7]. It can also be operating in water at temperatures up to $90^{\circ} \mathrm{C}$. A silent throttle valve in the test rig is used for the variation of 


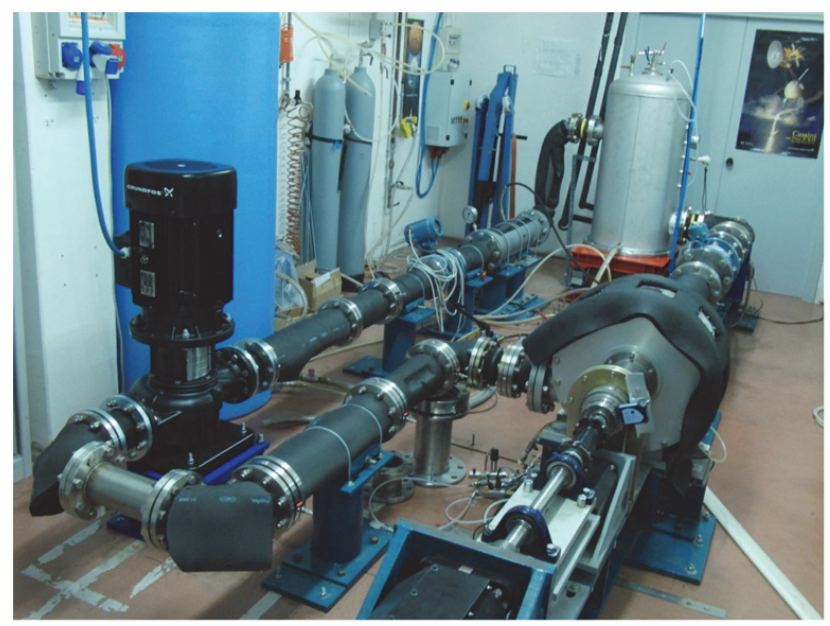

FIgure 4: The Cavitating Pump Rotordynamic Test Facility.

the pump load, whereas the constancy of the flow rate through the pump, especially useful in cavitating conditions, is obtained by means of an auxiliary pump (Grundfos TPE $100-390 / 2$ with a maximum pumping power of $22 \mathrm{~kW}$ at a rotating speed of $2920 \mathrm{rpm}$ ) mounted on the discharge line which is put in a feedback loop with the discharge flow meter. Two electromagnetic flow meters (mod. 8732E by FisherRosemount, range $0-100 \mathrm{l} / \mathrm{s}$, accuracy $0.5 \%$ FS), mounted on the suction and discharge lines of the water loop, provide the measurement of the inlet and outlet flow rates.

Figure 4 shows the configuration used for the experimental characterization of the DAPROT3 inducer with tip clearances of $0.8 \mathrm{~mm}$ and $2 \mathrm{~mm}$. In the configuration with the smaller tip blade clearance, the measurements of the pressure rise across the inducer have been taken as follows:

(a) The downstream pressure tap was located about two diameters downstream of the trailing edges of the inducer blades.

(b) The inlet pressure has been measured six diameters further upstream of the blade leading edge to consider possible prerotation effects of the inlet flow.

On the other hand, in the configuration with $2 \mathrm{~mm}$ tip blade clearance, the inlet pressure has been measured about two inducer diameters upstream of the blade leading edges, while the outlet pressure tap has been mounted on the discharge line at about 2.5 duct diameters downstream of the connection with the test chamber.

A pair of redundant differential pressure transducers measure the pump pressure rise between the inlet section and outlet section (Kulite, model BMD 1P 1500 100, $0 \div 6.8$ bar-d operating range, $0.1 \%$ precision class; Druck, model PMP $4170,0 \div 1$ bar-d operating range, $0.08 \%$ precision class). Hence, in this case, head measurements include both diffusion losses into the test section and entrance losses into the discharge line.

3.1. Test Item. The tested inducer, called DAPROT3, is a three-bladed high-head inducer with tapered hub and variable pitch and has been designed by means of a widely

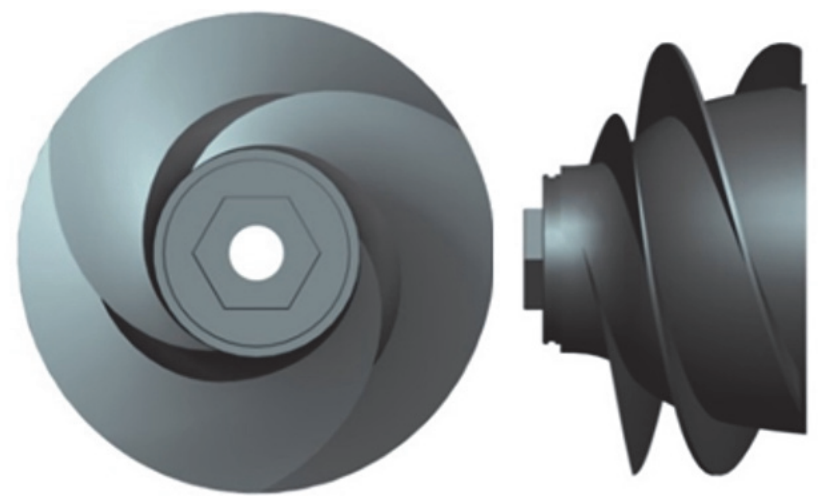

FIgURE 5: The DAPROT3 inducer made of 7075-T6 aluminum alloy with Sanford surface treatment.

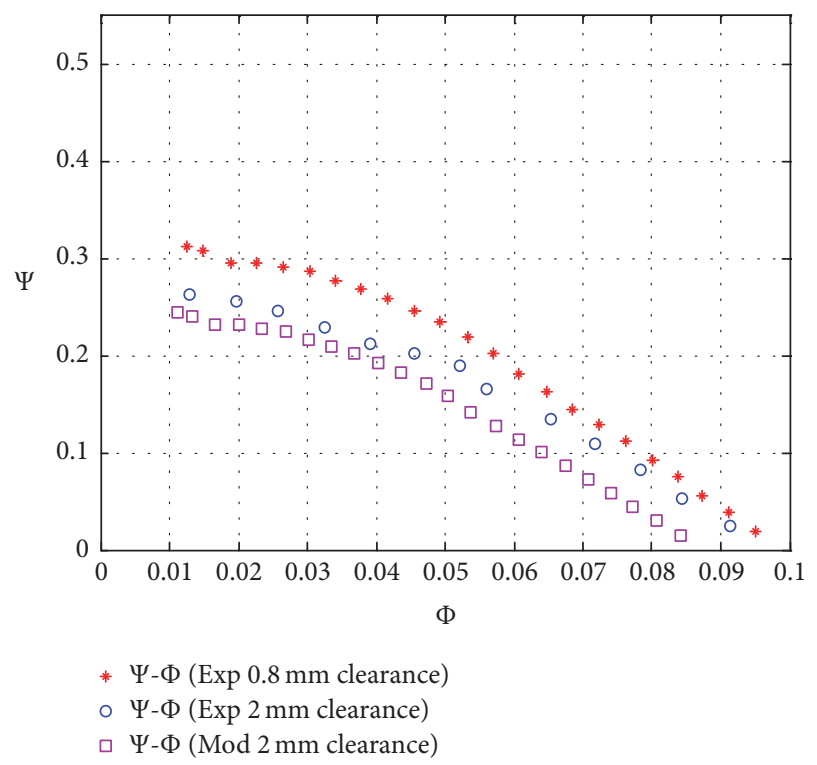

FIGURE 6: Hydraulic performances for the DAPROT3 inducer with different clearance values. For a clearance of $2 \mathrm{~mm}$, also the results of the model used for scaling the performance are shown.

validated reduced-order model developed by ALTA S.p.A. [13], as shown in Figure 5. The geometry generated by the model is consistent with the typical geometries and operational characteristics of modern space rocket inducers.

A series of tests conducted in water at room temperature $\left(20^{\circ} \mathrm{C}\right)$ have been carried out to assess the characterization of the pumping performances of this three-bladed axial inducer at different flow coefficients. All experiments have been carried out at Reynolds numbers $\left(\operatorname{Re}=2 \Omega r_{T}^{2} / \nu\right)$ higher than $10^{6}$ for the results to be virtually independent of turbulent effects, as confirmed by Brennen [14] in a series of tests at different rotating speeds. The pumping performance has been evaluated in terms of static head coefficients $(\Psi=$ $\left.\Delta p / \rho \Omega^{2} r_{T}^{2}\right)$, with the pressure rise measured at the locations as described above, as a function of the flow coefficient $(\Phi=$ $\left.\mathrm{Q} / \pi \Omega r_{T}^{3}\right)$.

The results of the experimental tests carried out on the two inducer configurations are shown in Figure 6, where they 


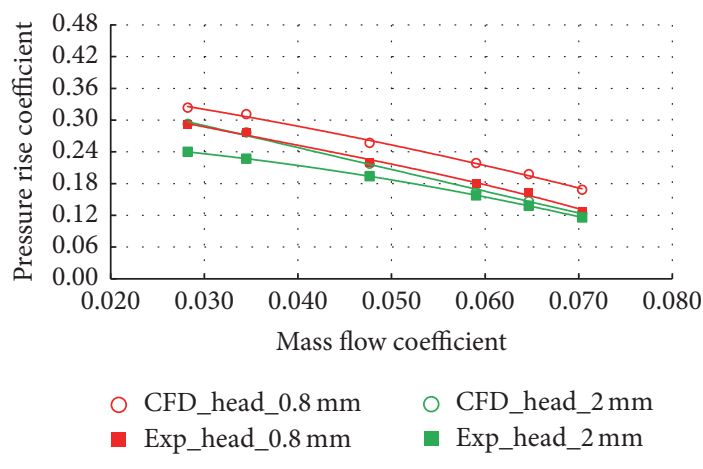

(a) $H$-Q curves

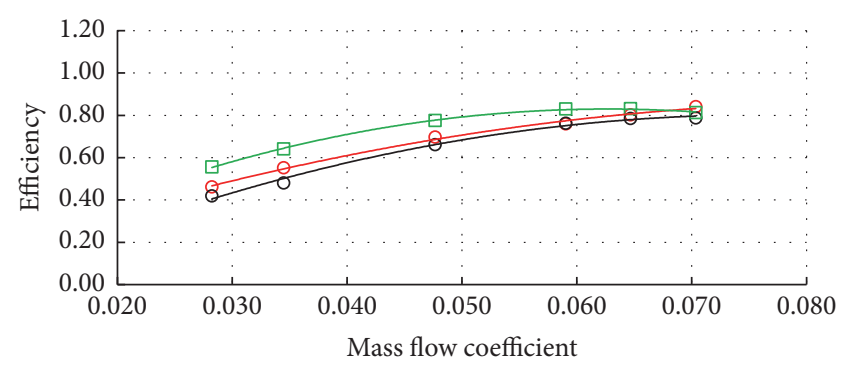

○ CFD_efficiency_0.8 mm O CFD_efficiency_2 $\mathrm{mm}$

$\square$ Exp_efficiency_2 $\mathrm{mm}$

(b) $\eta$-Q curves

Figure 7: Comparison of $H$ - $Q$ and $\eta$ - $Q$ curves based on different blade tip clearances.

are indicated by red crosses and blue dots for the $0.8 \mathrm{~mm}$ clearance and $2 \mathrm{~mm}$ clearance, respectively. Figure 6 also shows a third curve (pink squares) obtained by using a model developed and validated at ALTA for scaling the performance of inducers at different tip clearance values [3]. The model is based on the effects of tip clearance on both the flow coefficient and pump head. Starting from results obtained for $0.8 \mathrm{~mm}$ tip clearance, the model has been used for predicting the performance of the inducer operating with $2 \mathrm{~mm}$ blade tip clearance. They have been reported for later comparison with the numerical simulations, which have been carried out for two different tip clearances in the inducer configuration.

3.2. Test Results. The different intensity of tip clearance effects and also the position of the outlet pressure tap are thought to contribute to the observed discrepancy in the experimental hydraulic performance of the test inducer in the $0.8 \mathrm{~mm}$ and $2 \mathrm{~mm}$ tip clearance configurations. In the high clearance experiments the outlet pressure tap is located on the discharge line downstream of the test chamber, where the pressure reading is affected by flow diffusion losses into the test section and entrance losses into the discharge line. On the other hand, in low clearance experiments, the losses have been absent because the outlet pressure tap is located on the wall of the inducer casing about two diameters downstream of the blade trailing edges. However, at this location, the azimuthal flow velocity is not negligible and generates centrifugal effects capable of affecting the reading of the outlet static pressure of the inducer. Evaluating the azimuthal component by Carter's rule (as explained in detail in [15]), this influence decreases with the increase of the flow rate and so the two curves in Figure 6 tend to approach. Moreover, the readings of pressure rise in the two experimental configurations are also affected by flow acceleration/deceleration effects generated by the different cross sections of the flow at the measurement points. Also this aspect influences the static pressure at the downstream section in the direction of reducing the difference between the two curves as the flow rate increases. The above considerations are also fully consistent with the observation that the experimental results for $2 \mathrm{~mm}$ clearance (blue circles in Figure 6) are closer to the predictions of the inducer performance model (pink squares) at low flow rates, where tip clearance effects dominate, and tend to deviate more appreciably at high flow rates, where the influence of the different positions of the static pressure readings (not accounted in the model) is more significant.

\section{Results and Discussion}

4.1. Effects of Blade Tip Clearances on Hydraulic Performances. As the hydraulic performances of the inducer with the blade tip clearances of $0.8 \mathrm{~mm}$ and $2 \mathrm{~mm}$ were experimentally analyzed above, the influences of the tip blade clearances were also numerically investigated. Meanwhile, the pumping performances of the inducer with the blade tip clearances of $0.8 \mathrm{~mm}$ and $2 \mathrm{~mm}$ were further predicted and compared with the corresponding experimental results.

As expected, as shown in Figure 7(a), the pressure difference between the inducer inlet and the outlet is larger when the blade clearance is smaller. Meanwhile, a higher blade clearance implies a decrease in the pump performances obtained from both experimental and numerical data. It is probably the reason that large loss was found in the inducer with higher blade tip clearance.

With respect to the hydraulic efficiency of the inducer, as shown in Figure 7(b), the Efficiency-Mass Flow curve of the inducer with the larger blade clearance is obviously lower than that of the inducer with smaller blade clearance especially in low flow rates. However, with the flow rate increasing, the effect of the blade clearance on the hydraulic efficiencies turns small, and the three Efficiency-Mass Flow curves form a similar shape and finally become almost coincident with each other especially near the design operating conditions.

In addition, it can be also found that the hydraulic efficiencies of the inducer with the higher blade clearance of $2 \mathrm{~mm}$ predicted by CFD have a good agreement with those obtained from experiment.

4.2. Analysis of Internal Flow in the Inducer. Based on the above analysis of the pump performances of the inducer under a wide range of flow rates, it can be obtained that the blade clearance has a significant influence on the static pressure rise and hydraulic efficiency especially when the inducer runs near the low flow rates. As the clearance between the blade tip and the inducer casing becomes larger, the hydraulic diffusion loss increases and also the induced 


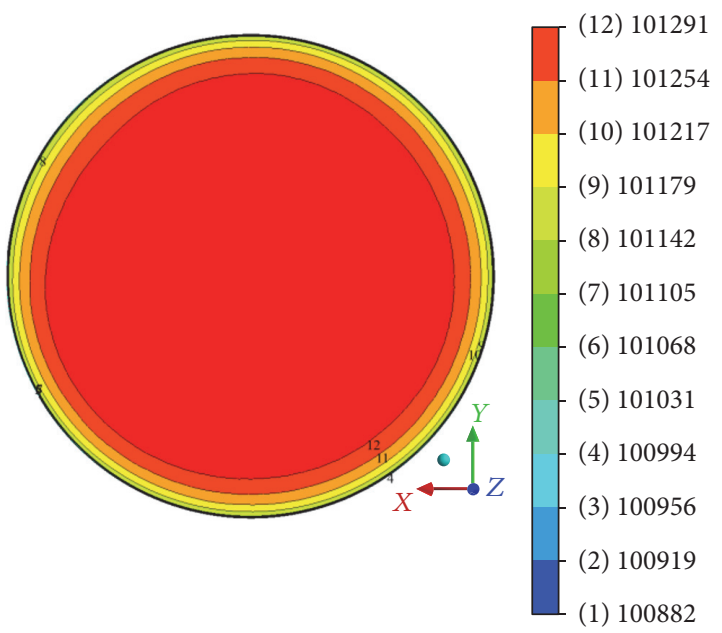

(a)

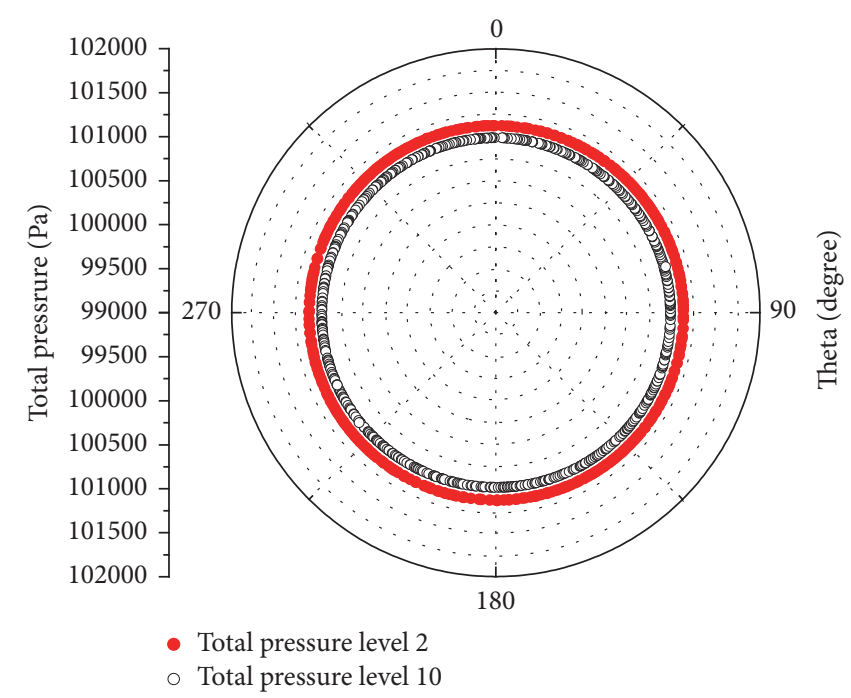

(b)

FIGURE 8: Distributions of the total pressure (a) and total pressure level 2 and level 10 (b) in the theta direction at $1.0 Q_{d}$.

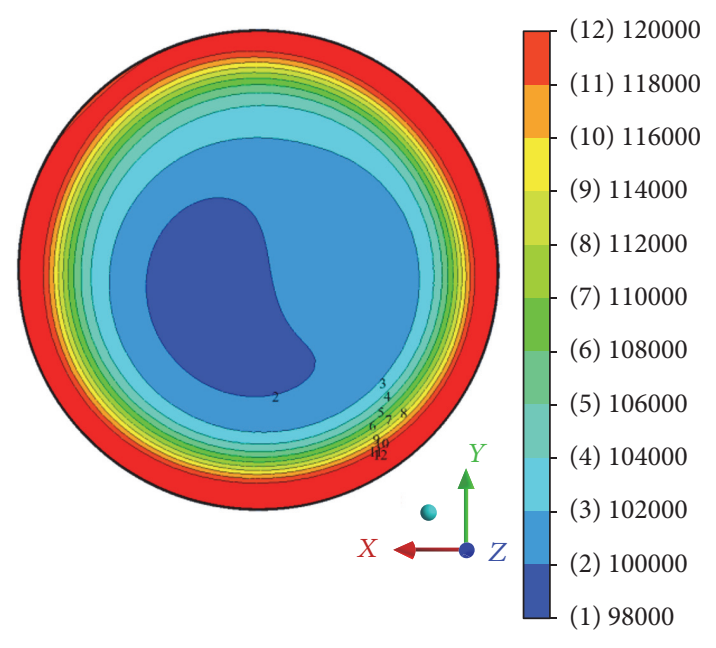

(a)

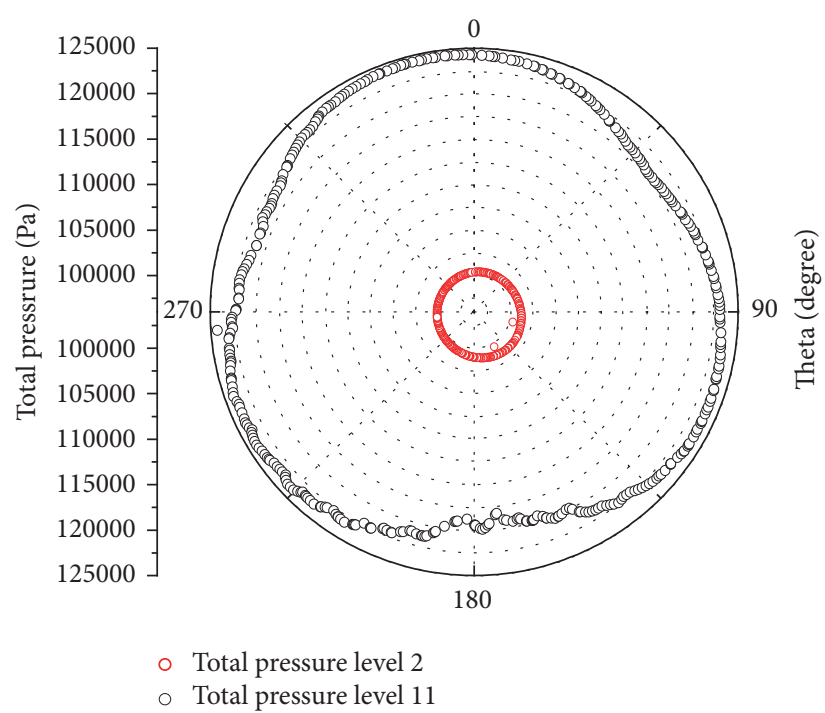

(b)

FIGURE 9: Distributions of the total pressure (a) and total pressure level 2 and level 11 (b) in the theta direction at $0.4 Q_{d}$.

backflow in the inducer inlet at low flow rates becomes stronger and has a negative effect in the main flow in the inlet pipe.

Meanwhile, the flow patterns at low flow rates are very complex and are always associated with backflow in the inlet pipe which may cause flow instabilities or even cavitation instabilities in the inducer. Therefore, the internal flow in the inducer with the smaller clearance under design and offdesign operating conditions was especially investigated in present simulations. The high, design, and low flow rates are $110 \%, 100 \%$, and $40 \%$ of the design flow rate, respectively.

4.3. Total Pressure Distribution in the Inlet Duct. The total pressure near and far away from the center of the inlet pipe was specially analyzed such as isobar line 2 and line 11 with respect to the total pressure contour lines. The cross section plane was made based on position 1 (one diameter upstream of the blade leading edge for the inlet station and one diameter downstream of the blade trailing edge for the outlet station, corresponding to the locations where the inlet and outlet pressure transducers have been installed in the experimental tests on the inducer with $0.8 \mathrm{~mm}$ blade tip clearance.). The total pressure in the radial and theta direction under $1.0 Q_{d}$, the design flow rate, and $0.4 Q_{d}$ were investigated. The rotational direction of the inducer was counterclockwise.

As shown in Figure 8, at the design flow rate, the total pressure in this plane shows a uniform distribution, even though the total pressure is a little higher in the central part of the inlet pipe than that near the pipe wall. In particular, 


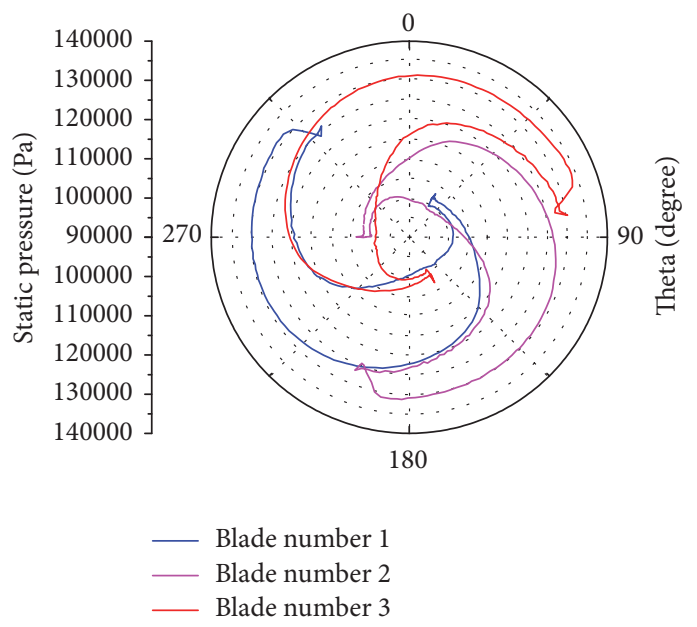

(a)

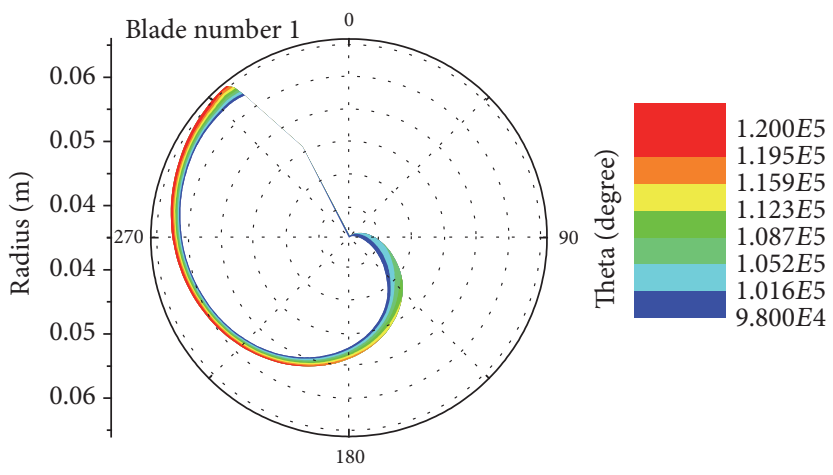

b(i)

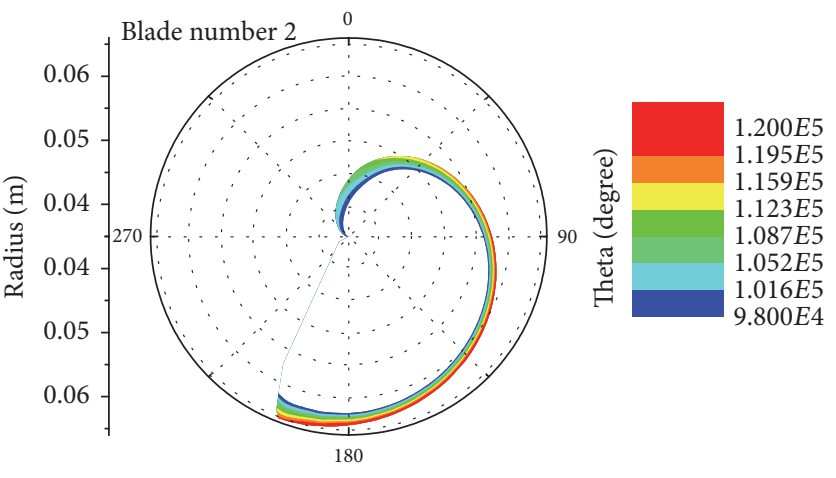

b(ii)

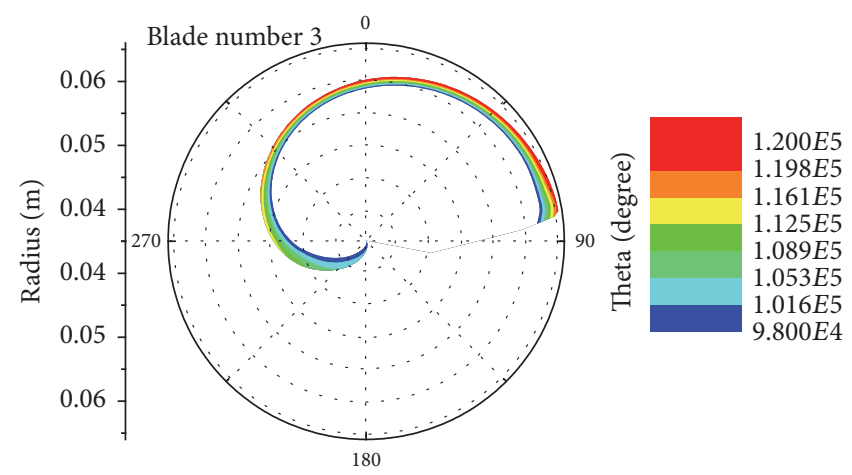

b(iii)

(b) The contour of static pressure distribution on each blade

FIGURE 10: Static pressure distributions and pressure load on the blades of the inducer at $1.1 Q_{d}$.

in the theta direction along with the rotational direction of the inducer, the total pressure shows a concentric circle with the corresponding constant radius as for contour line 2 and contour line 10, respectively, as shown in this figure.

At the flow rate of $0.4 Q_{d}$, as shown in Figure 9, a significantly low total pressure core occurs in contour line 2 , and the total pressure increases from the pipe center to the pipe wall. There is a big pressure gradient between contour lines 2 and 11. Moreover, along the counterclockwise direction, the total pressure distribution shows an irregular circle with variable radius as for contour line 11 where there are three high total pressure regions.

Therefore, the flow characteristics between the design flow rate and low flow rates are totally different. It is probably reversed flows that occur and influence the main flow in the inlet pipe at the low flow rate.

4.4. Static Pressure Distribution and Pressure Load on Each Blade of the Inducer. In order to further investigate the internal flow in the inducer, the static pressure distribution on 


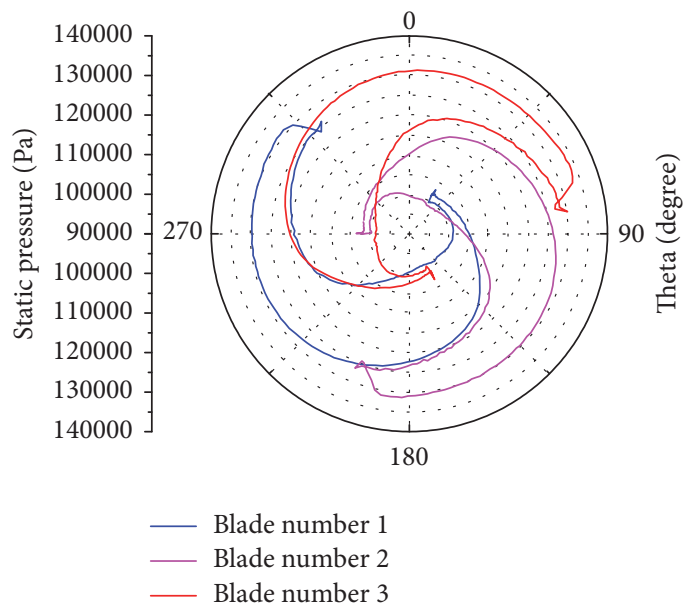

(a)

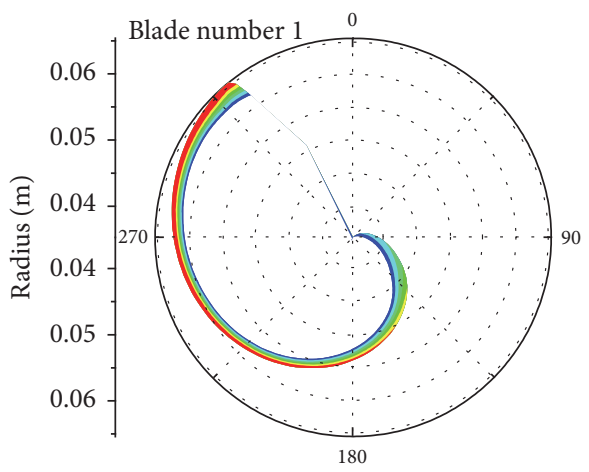

b(i)

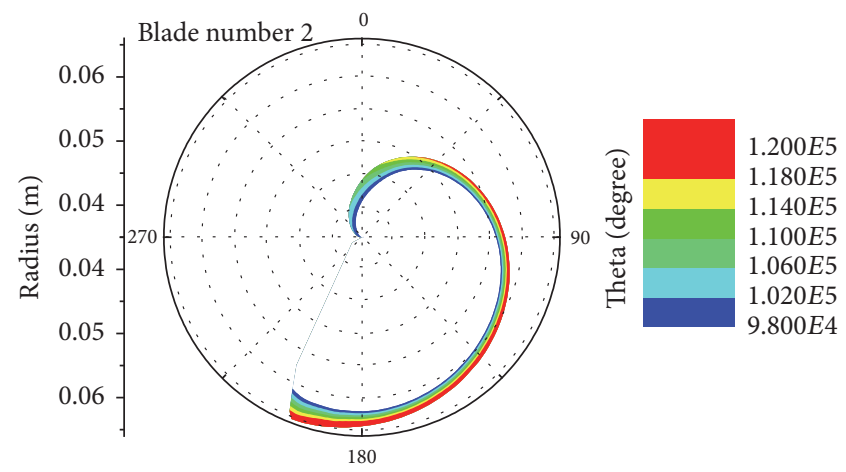

b(ii)

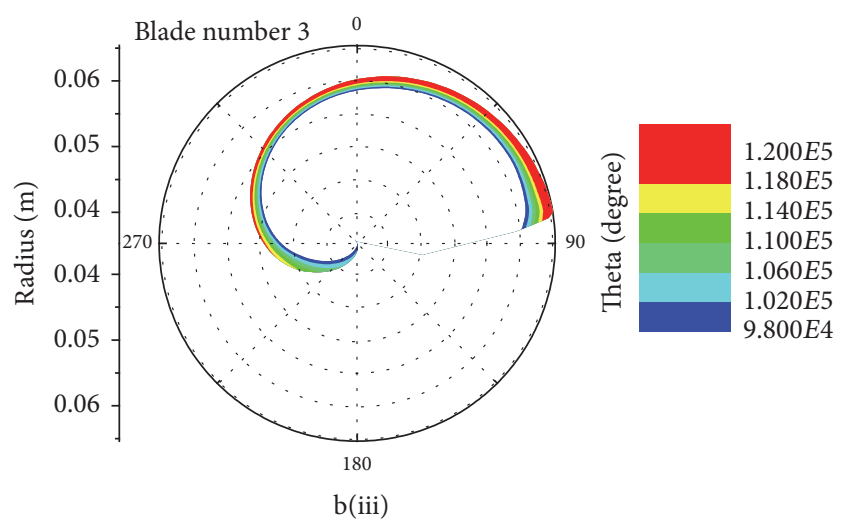

(b) The contour of static pressure distribution on each blade

FIGURE 11: Static pressure distributions and pressure load on the blades of the inducer at $1.0 Q_{d}$.

each blade suction side and pressure side was obtained. The pressure load on the blades in the theta and radial directions was also analysed.

As shown in Figures 10,11, and 12, the static pressure distribution on the three blades was uniform and the pressure load formed almost the same pattern like the blade shape in the theta direction for the high flow rate of $1.1 Q_{d}$, the design flow rate of $1.0 Q_{d}$, and the low flow rate of $0.4 Q_{d}$. The values of static pressure are almost constant from blade to blade for the same flow rate. However, static pressure values in each blade pressure side are higher than that in the blade suction side, and the pressure differences between the blade suction side and the pressure side decrease from each blade inlet to the outlet.

In addition, the pressure load on the blades was also investigated for high flow rate $\left(1.1 Q_{d}\right)$, design flow rate $\left(1.0 Q_{d}\right)$, and low flow rate $\left(0.4 Q_{d}\right)$. As shown in Figure 13(a), the pressure rise coefficients are almost constant for the same blade inlet at the three different flow rates. However, at the flow coefficient of $0.4 Q_{d}$, the pressure rise coefficient in the 


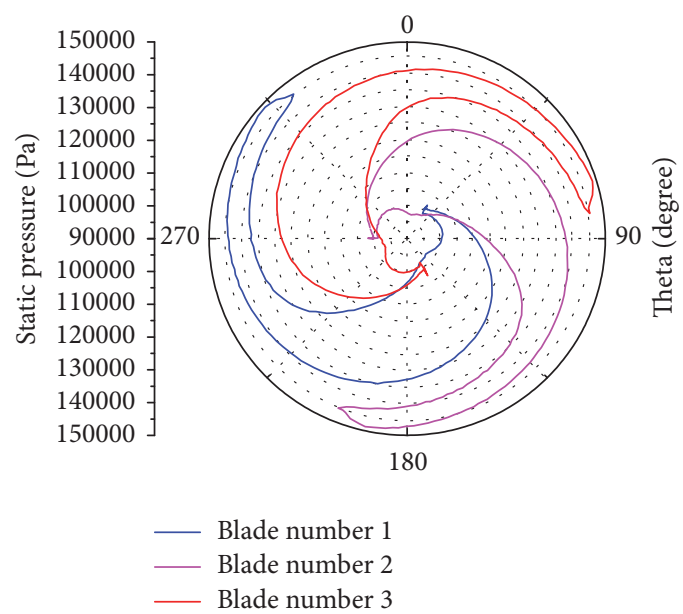

(a)

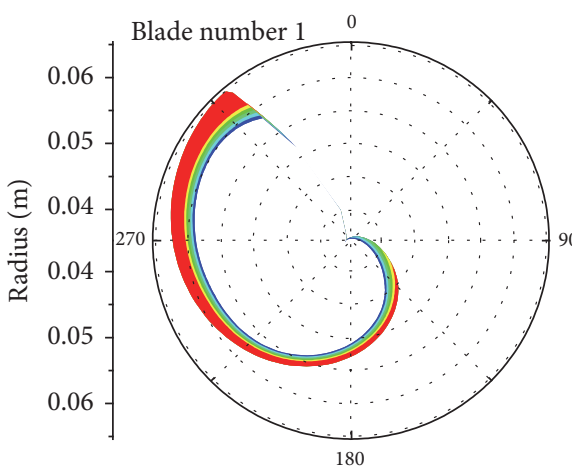

b(i)

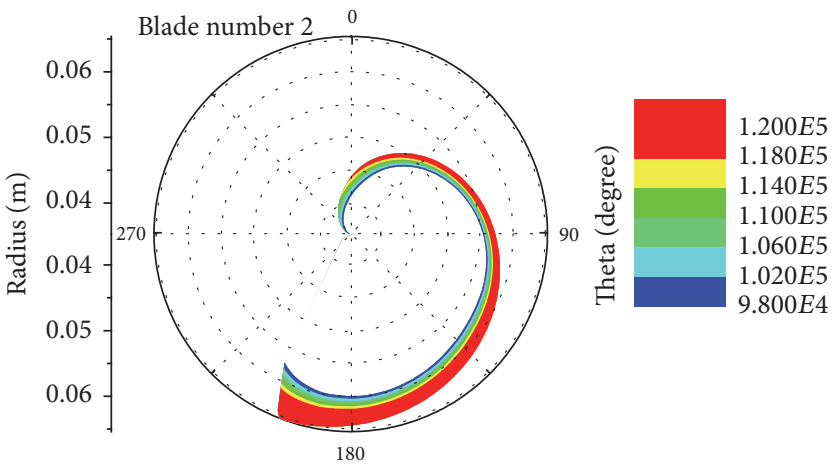

b(ii)

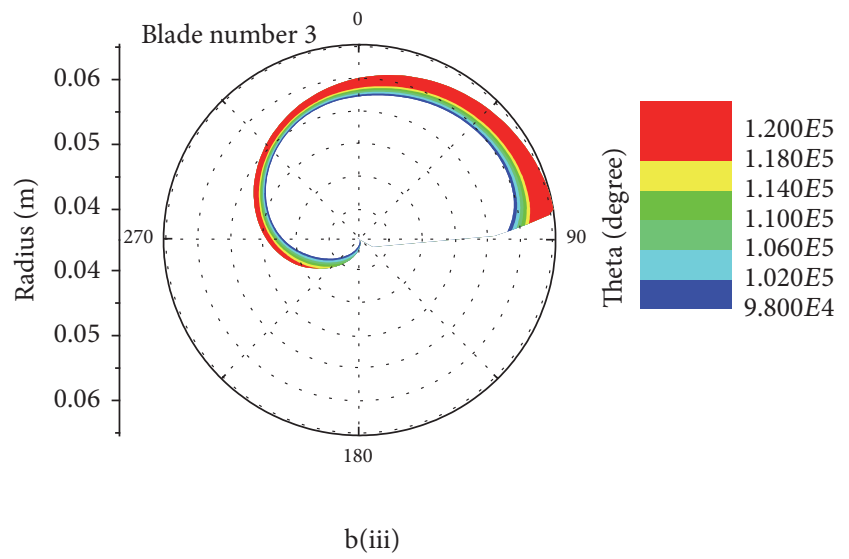

(b) The contour of static pressure distribution on each blade

FIGURE 12: Static pressure distributions and pressure load on the blades of the inducer at $0.4 Q_{d}$.

blade outlet becomes much larger than those in design and high flow rates.

Figure 13(b) also shows the static pressure distributions in blade radial direction under the high, design, and low flow rates. In consistency with the results of Figure 13(a), the static pressure distributions in the blade radial direction show almost the same patterns like each blade shape with pressure load increasing as the radius increases. The pressure rise is much larger at low flow rate of $0.4 Q_{d}$ than those in design and high flow rates.

\section{Conclusions}

The numerical hydraulic performances of the inducer with the blade tip clearances of $0.8 \mathrm{~mm}$ and $2 \mathrm{~mm}$ have been mainly analyzed in comparison with the experimental results. 


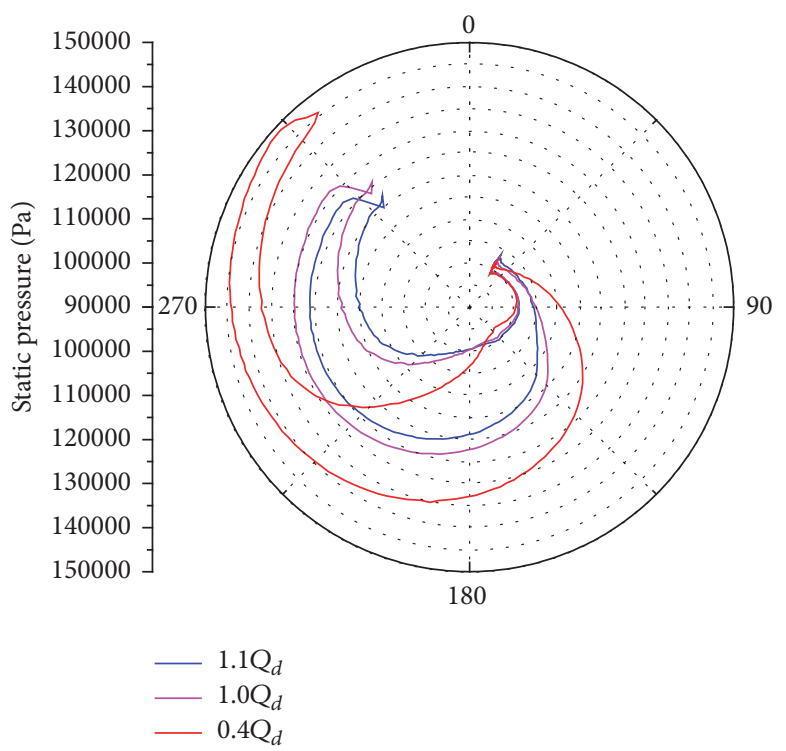

(a)

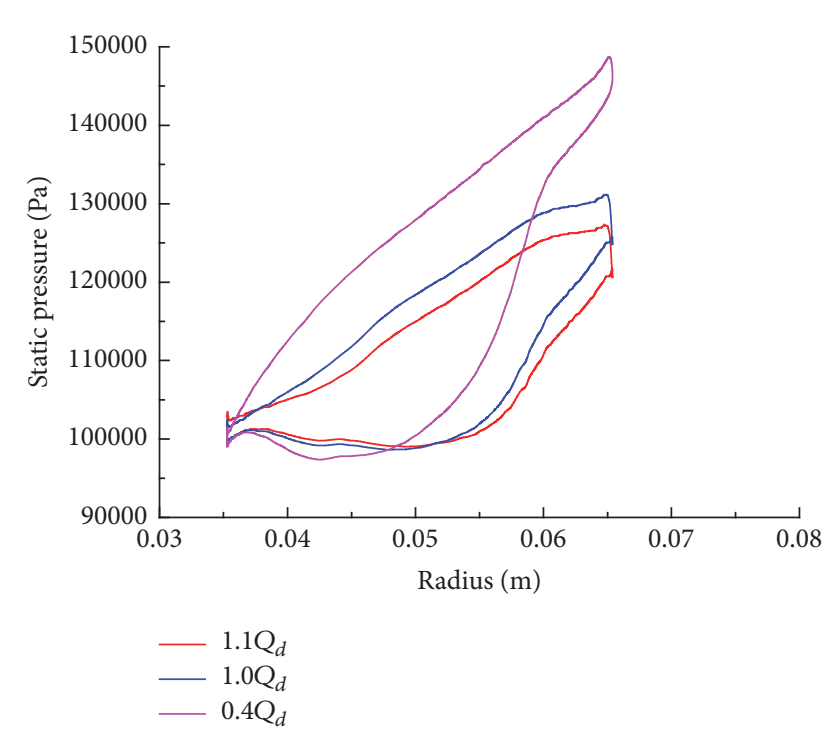

(b)

FIGURE 13: Comparison of static pressure distributions on the blades in the theta direction (a) and the blade radial direction (b) at the flow rates of $1.1 Q_{d}$ and $1.0 Q_{d}$ and $0.4 Q_{d}$.

Moreover, the internal flow patterns of the inducer at design and off-design flow rates were further investigated in present simulations. From the analysis of the results, the following conclusions have been drawn:

(1) The more refined mesh was more capable of modeling the internal flow and pump performances in the inducer satisfactorily. The inducer with the smaller blade tip clearance of $0.8 \mathrm{~mm}$ has the characteristics of higher pressure rise than that with the lager blade tip clearance of $2 \mathrm{~mm}$.

(2) The blade clearance has a significant influence on the static pressure rise and hydraulic efficiency especially when the inducer runs near the low flow rates.

(3) The larger clearance between the blade tip and the inducer casing causes larger hydraulic diffusion loss and also induces stronger backflow in the inducer inlet at low flow rates.

In summary, the good agreement in the hydraulic performances of the inducer obtained between the experimental and numerical results of the present research activity indicates that the proposed numerical methods can adequately capture the internal flow and the related hydrodynamic effects in the test inducer. The model and CFD methods can therefore be used as an effective tool to understand, analyze, predict, and control the mechanisms of the complex phenomena taking place in the inner flows in inducers operating especially at low flow rates.

\section{Nomenclature}

c: Tip blade clearance, $\mathrm{m}$

$D$ : Diffusion factor
$L:$ Axial length, $\mathrm{m}$

$N$ : Number of blades

$\Omega$ : Inducer rotational speed, $\mathrm{rad} / \mathrm{s}$

$p$ : Static pressure, $\mathrm{Pa}$

Q: Volumetric flow rate, $\mathrm{m} 3 / \mathrm{h}$

$\Phi$ : Flow coefficient

$\Psi$ : Static head coefficient

$\delta$ : Tip clearance

$\gamma$ : Blade angle from axial direction

$\rho$ : Liquid density

$s:$ Azimuthal blade spacing

$\sigma$ : Blade solidity $=c / s$.

\section{Subscripts}

BLE: Blade leading edge

BTE: Blade trailing edge

BTP: Blade tip

BS: Blade suction

BP: Blade pressure.

\section{Competing Interests}

The authors declare that there is no conflict of interests regarding the publication of this paper.

\section{Acknowledgments}

This study is supported by National Natural Science of China (no. 51609107 and no. BK20160539), Jiangsu University Senior Personnel Scientific Research Foundation (no. 15JDG073), China Postdoctoral Science Foundation (no. 2014M561581), the Open Research Subject of Key Laboratory (Research Base) of Key Laboratory of Fluid and Power Machinery, 
Ministry of Education (no. szjj2016-065), and the Priority Academic Program Development of Jiangsu Higher Education Institutions. The present DAPROT3 experimental work has been carried out in ALTA under ESA's support (no. 4000102585/10/NL/Sfe).

\section{References}

[1] C. E. Brennen, "A review of the dynamics of cavitating pumps," IOP Conference Series: Earth and Environmental Science, vol. 15, no. 1, Article ID 012001, 2012.

[2] J. H. Kim and A. J. Acosta, "Unsteady flow in cavitating turbopumps," ASME Journal of Fluids Engineering, vol. 97, no. 4, pp. 412-417, 1975.

[3] L. Torre, A. Pasini, A. Cervone, G. Pace, P. Miloro, and L. d'Agostino, "Effect of tip clearance on the performance of a three-bladed axial inducer," Journal of Propulsion and Power, vol. 27, no. 4, pp. 890-898, 2011.

[4] K. Okita, H. Ugajin, and Y. Matsumoto, "Numerical analysis of the influence of the tip clearance flows on the unsteady cavitating flows in a three-dimensional inducer," Journal of Hydrodynamics, vol. 21, no. 1, pp. 34-40, 2009.

[5] Y. Tsujimoto, "Simple rules for cavitation instabilities in turbomachinery," in Proceedings of the Symposium on Cavitation, Invited Lecture, Pasadena, Calif, USA, 2001.

[6] F. Bakir, R. Rey, A. Gerber, T. Belamri, and B. Hutchinson, "Numerical and experimental investigations of the cavitating behavior of an inducer," The International Journal of Rotating Machinery, vol. 10, pp. 15-25, 2004.

[7] E. Rapposelli, A. Cervone, and L. d'Agostino, "A new cavitating pump rotordynamic test facility," in Proceedings of the 38th AIAA/ASME/SAE/ASEE Joint Propulsion Conference and Exhibit 2002, Indianapolis, Ind, USA, July 2002.

[8] G. Pace, D. Valentini, A. Pasini, L. Torre, Y. Fu, and L. D’Agostino, "Geometry effects on flow instabilities of different three-bladed inducers," Journal of Fluids Engineering, Transactions of the ASME, vol. 137, no. 4, pp. 1-12, 2015.

[9] Y. Fu, Y. Fang, J. Yuan, S. Yuan, G. Pace, and L. Dagostino, "Study on hydraulic performances of a 3-bladed inducer based on different numerical and experimental methods," International Journal of Rotating Machinery, vol. 2016, Article ID 4267429, 9 pages, 2016.

[10] B. E. Launder and D. B. Spalding, "The numerical computation of turbulent flows," Computer Methods in Applied Mechanics and Engineering, vol. 3, no. 2, pp. 269-289, 1974.

[11] H. Ding, F. C. Visser, Y. Jiang, and M. Furmanczyk, "Demonstration and validation of a 3D CFD simulation tool predicting pump performance and cavitation for industrial applications," ASME Journal of Fluids Engineering, vol. 133, no. 1, 14 pages, 2011.

[12] O. Coutier-Delgosha, R. Fortes-Patella, and J. L. Reboud, "Evaluation of the turbulence model influence on the numerical simulations of unsteady cavitation," Journal of Fluids Engineering, vol. 125, no. 1, pp. 38-45, 2003.

[13] D. Valentini, A. Pasini, G. Pace, L. Torre, and L. D’Agostino, "Experimental validation of a reduced order for radial turbopump design," in Proceedings of the 49th AIAA/ASME/SAE/ASEE Joint PropulsionConference, AIAA, San Jose, Calif, USA, July 2013.

[14] C. E. Brennen, Hydrodynamics of Pumps, Concepts ETI, Inc., Norwich, Vt, USA; Oxford University Press, Oxford, UK, 1994.
[15] C. E. Brennen, Cavitation and Bubble Dynamics, Oxford University Press, Oxford, UK, 1995. 


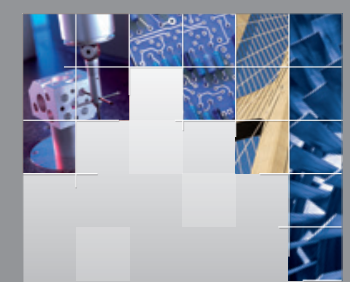

\section{Enfincering}
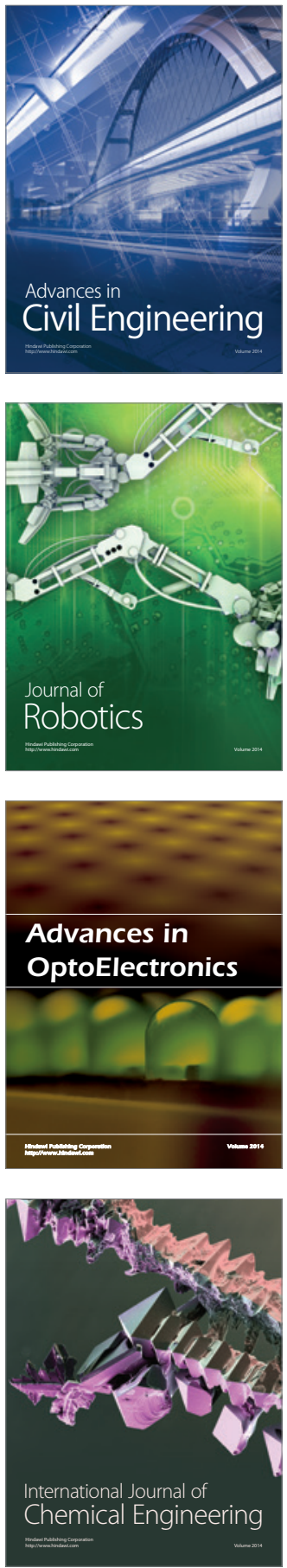

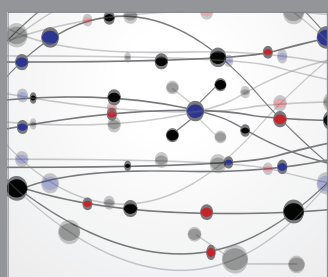

The Scientific World Journal

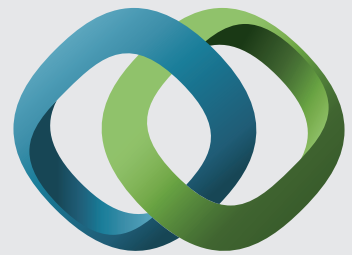

\section{Hindawi}

Submit your manuscripts at

https://www.hindawi.com
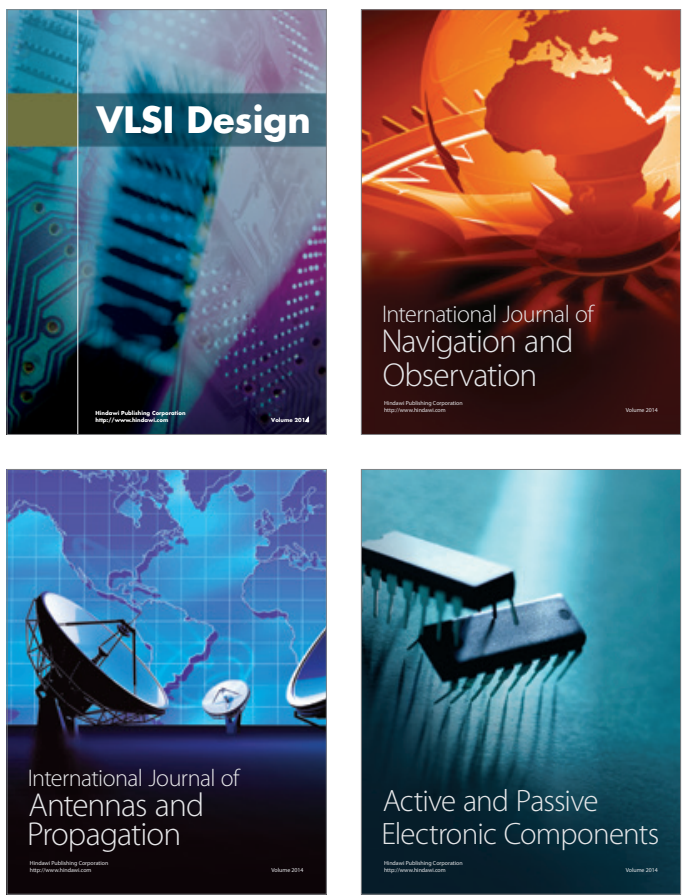
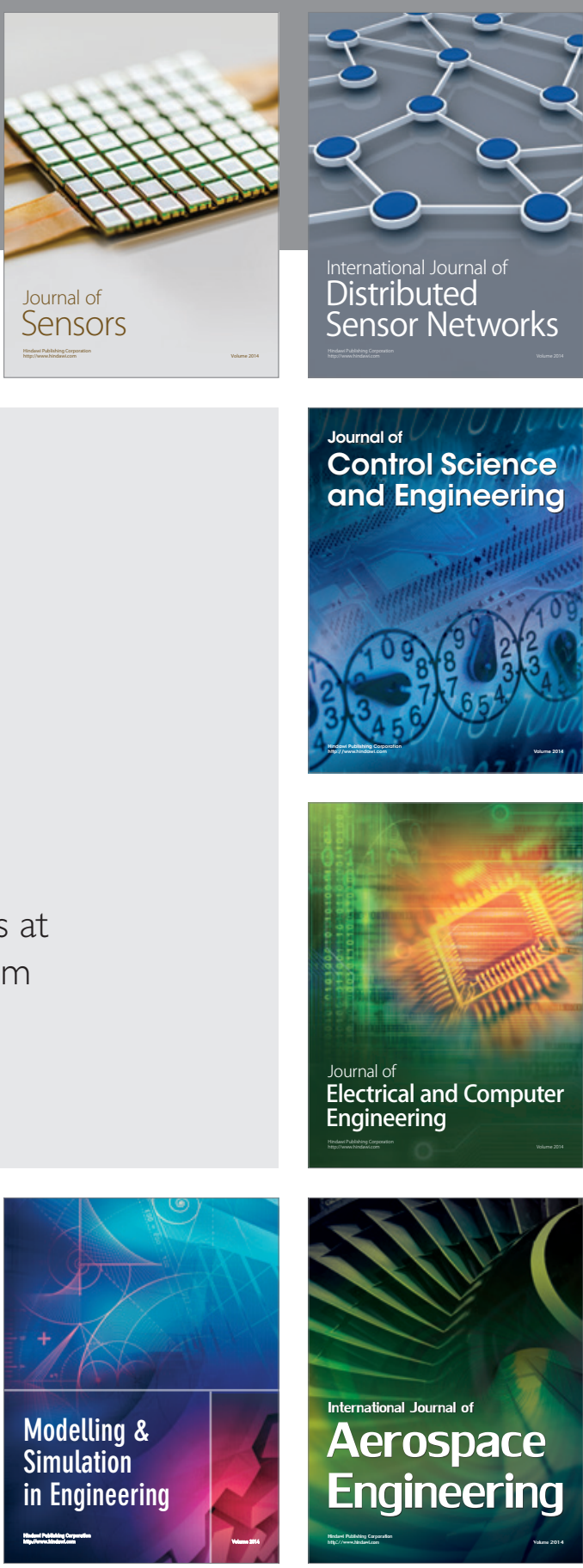

International Journal of

Distributed

Sensor Networks

$-$

Joumal of

Control Science

and Engineering
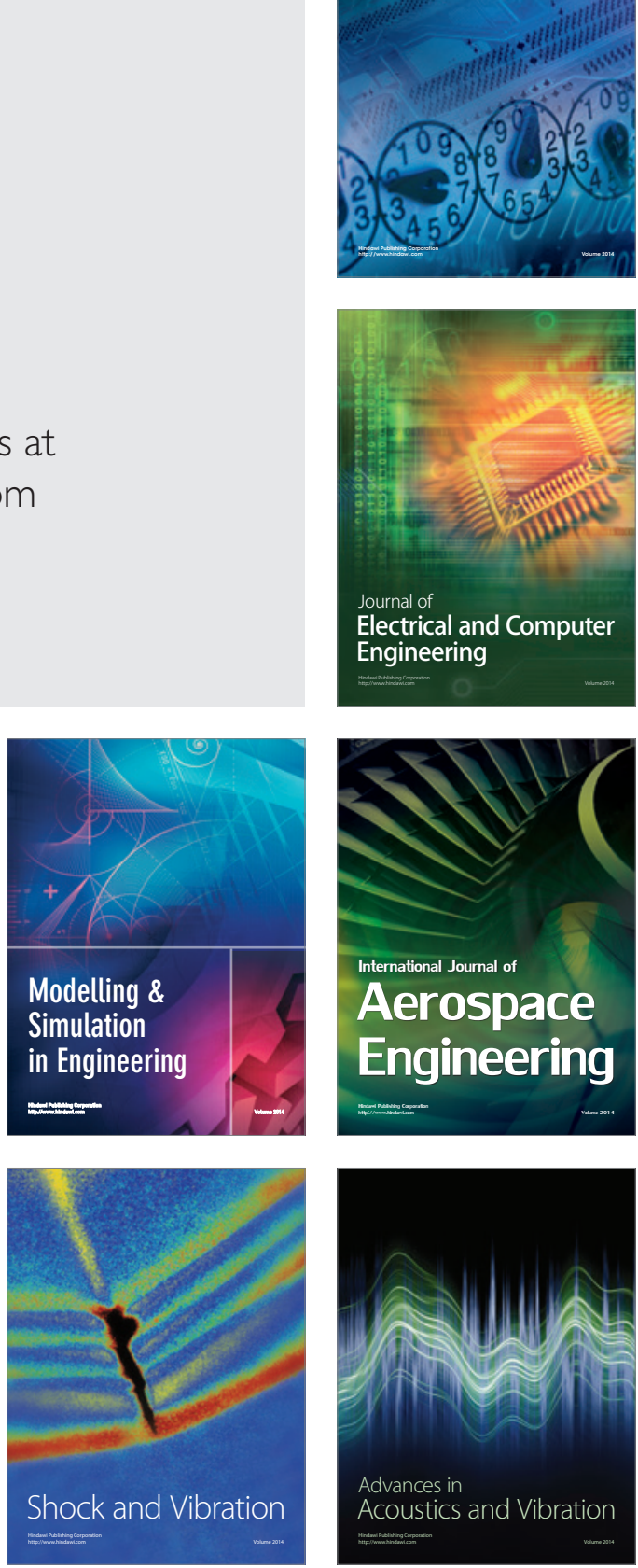